\begin{tabular}{|c|l|}
\hline Title & $\begin{array}{l}\text { Structure analysis of geranyl pyrophosphate methyltransferase and the proposed reaction mechanism of SAM- } \\
\text { dependent C-methylation }\end{array}$ \\
\hline Author(s) & $\begin{array}{l}\text { A riyawutthiphan, Orapin; Ose, Toyoy uki; Minami, A tsushi; Sinde, Sandip; Tsuda, Muneya; Gao, Y ong-Gui; Y ao, Min; } \\
\text { Oikawa, Hideaki; Tanaka, Isao }\end{array}$ \\
\hline Citation & $\begin{array}{l}\text { Acta Crystallographica Section D: Biological Crystall lography, 68(11), 1558 1569 } \\
\text { https://doi.org/10.1107/S0907441912038486 }\end{array}$ \\
\hline Issue Date & 2012-11 \\
\hline Doc URL & http://hdl.handle.net/2115/50689 \\
\hline Type & article \\
\hline File Information & ACD68_11_1558_1569.pdf \\
\hline
\end{tabular}

Instructions for use 
Acta Crystallographica Section D

Biological

Crystallography

ISSN 0907-4449

Editors: E. N. Baker and Z. Dauter

\title{
Structure analysis of geranyl pyrophosphate methyltransferase and the proposed reaction mechanism of SAM-dependent C-methylation
}

\author{
Orapin Ariyawutthiphan, Toyoyuki Ose, Atsushi Minami, Sandip Sinde, \\ Muneya Tsuda, Yong-Gui Gao, Min Yao, Hideaki Oikawa and Isao Tanaka
}

Acta Cryst. (2012). D68, 1558-1569

Copyright (C) International Union of Crystallography

Author(s) of this paper may load this reprint on their own web site or institutional repository provided that this cover page is retained. Republication of this article or its storage in electronic databases other than as specified above is not permitted without prior permission in writing from the IUCr.

For further information see http://journals.iucr.org/services/authorrights.html

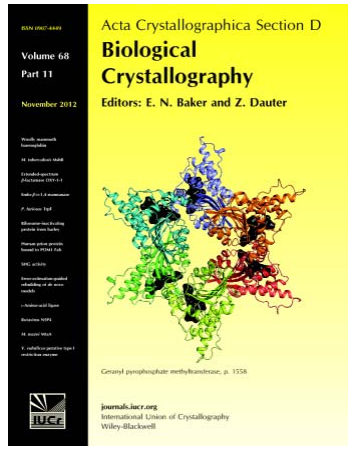

\begin{abstract}
Acta Crystallographica Section D: Biological Crystallography welcomes the submission of papers covering any aspect of structural biology, with a particular emphasis on the structures of biological macromolecules and the methods used to determine them. Reports on new protein structures are particularly encouraged, as are structure-function papers that could include crystallographic binding studies, or structural analysis of mutants or other modified forms of a known protein structure. The key criterion is that such papers should present new insights into biology, chemistry or structure. Papers on crystallographic methods should be oriented towards biological crystallography, and may include new approaches to any aspect of structure determination or analysis. Papers on the crystallization of biological molecules will be accepted providing that these focus on new methods or other features that are of general importance or applicability.
\end{abstract}

Crystallography Journals Online is available from journals.iucr.org 
Acta Crystallographica Section D

Biological

Crystallography

ISSN 0907-4449

Orapin Ariyawutthiphan, ${ }^{a}$

Toyoyuki Ose, ${ }^{b_{*}}$ Atsushi

Minami, ${ }^{c}$ Sandip Sinde, ${ }^{c}$ Muneya

Tsuda," Yong-Gui Gao,' Min

Yao, ${ }^{b}$ Hideaki Oikawa ${ }^{c}$ and

Isao Tanakad

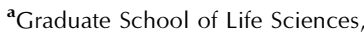
Hokkaido University, N10 W8 Kita-Ku, Sapporo 060-0810, Japan, ${ }^{\mathbf{b}}$ Faculty of Pharmaceutical Sciences, Hokkaido University, N10 W8 Kita-Ku, Sapporo 060-0810, Japan, 'Division of Chemistry, Graduate School of Science, Hokkaido University, N10 W8 Kita-Ku, Sapporo 060-0810, Japan, and ${ }^{\mathbf{d}}$ Faculty of Advanced Life Sciences, Hokkaido University, N10 W8 Kita-Ku, Sapporo 060-0810, Japan

Correspondence e-mail:

ose@pharm.hokudai.ac.jp

\section{Structure analysis of geranyl pyrophosphate methyltransferase and the proposed reaction mechanism of SAM-dependent $C$-methylation}

In the typical isoprenoid-biosynthesis pathway, condensation of the universal $\mathrm{C}_{5}$-unit precursors isopentenyl pyrophosphate (IPP) and dimethylallyl pyrophosphate (DMAPP) occurs via the common intermediates prenyl pyrophosphates $\left(\mathrm{C}_{10}-\mathrm{C}_{20}\right)$. The diversity of isoprenoids reflects differences in chain length, cyclization and further additional modification after cyclization. In contrast, the biosynthesis of 2-methylisonorneol (2-MIB), which is responsible for taste and odour problems in drinking water, is unique in that it primes the enzymatic methylation of geranyl pyrophosphate (GPP) before cyclization, which is catalyzed by an $S$-adenosyl-L-methioninedependent methyltransferase (GPPMT). The substrate of GPPMT contains a nonconjugated olefin and the reaction mechanism is expected to be similar to that of the steroid methyltransferase (SMT) family. Here, structural analysis of GPPMT in complex with its cofactor and substrate revealed the mechanisms of substrate recognition and possible enzymatic reaction. Using the structures of these complexes, methyl-group transfer and the subsequent proton-abstraction mechanism are discussed. GPPMT and SMTs contain a conserved glutamate residue that is likely to play a role as a general base. Comparison with the reaction mechanism of the mycolic acid cyclopropane synthase (MACS) family also supports this result. This enzyme represented here is the first model of the enzymatic $C$-methylation of a nonconjugated olefin in the isoprenoid-biosynthesis pathway. In addition, an elaborate system to avoid methylation of incorrect substrates is proposed.

\section{Introduction}

Natural isoprenoids consist of terpenoids, steroids, carotenoids, prenylated quinones (menaquinone) and dolichol phosphate (a sugar carrier), and may include prenylated proteins. These are synthesized by prenylation using the universal $\mathrm{C}_{5}$-unit precursors isopentenyl pyrophosphate (IPP) and dimethylallyl pyrophosphate (DMAPP) via common intermediates: the prenyl pyrophosphates $\left(\mathrm{C}_{10}-\mathrm{C}_{20}\right)$. The diversity of isoprenoids originates from chain length and cyclization modes. Further diversification of isoprenoids is effected by oxidative modifications and the introduction of various groups, such as by acylation, glycosylation and alkylation. In addition to these modifications, C1-methylation of the prenyl chain as in the case of the steroid side chain adds further diversity to the isoprenoids. Recently, unprecedented methylation of geranyl pyrophosphate (GPP) was found in the biosynthesis of the bacterial homo-monoterpene 2-methylisoborneol (2-MIB).

2-MIB, which is widely produced by actinomycetes (Medsker et al., 1969), cyanobacteria (Giglio et al., 2011) and
Received 13 July 2012

Accepted 7 September 2012

PDB References: geranyl pyrophosphate methyltransferase, complex with SAM, 4f84; apo, 4f85; complex with GPP and sinefungin, $4 \mathrm{f} 86$ 
myxobacteria (Dickschat et al., 2007), is responsible for an unpleasant musty odour, and its odorous threshold is below $10 \mathrm{ng}^{-1}$ (Young et al., 1996). The occurrence of 2-MIB in drinking water is a common and widespread problem in water supplies. In a biosynthetic study of 2-MIB, labelling experiments confirmed that the 'extra' C2 methyl group is derived from $S$-adenosyl-L-methionine (SAM; Bentley \& Meganathan, 1981). The involvement of 2-methylgeranyl pyrophosphate (2-methyl-GPP) was proposed, based on feeding experiments in the myxobacterium Nannocystis exedens, indicating that the SAM-dependent methylation of GPP proceeds prior to cyclization (Dickschat et al., 2007). Bioinformatics approaches based on hidden Markov models (Eddy, 1998) enabled the identification of a putative operon encoding GPP methyltransferase (GPPMT) and 2-MIB synthase in various actinomycetes (Komatsu et al., 2008). The production of 2-MIB by heterologous expression of these translationally coupled genes from various bacteria using Streptomyces avermitilis, which is naturally deficient in 2-MIB biosynthesis, as a host (Komatsu et al., 2008) was confirmed (Fig. 1). Recently, we reported that recombinant GPP methyltransferase (GPPMT) and monoterpene synthase from $S$. lasaliensis convert GPP to 2-MIB via 2-methyl-GPP (Komatsu et al., 2008). This conversion by these two proteins (expressed in Escherichia coli) from $S$. coelicolor A3(2) (Wang \& Cane, 2008) as well as S. lasaliensis (Komatsu et al., 2008) has also been shown.

SAM-dependent methyltransferases (MTs) catalyze various $\mathrm{C}_{1}$-transfer reactions. Among them, enzymatic $C$-methylations of macromolecules such as DNA and RNA have been studied extensively and their detailed mechanisms have been established. SAM-MTs also catalyze $C$-methylation of a number of secondary metabolites, which are biosynthesized via methylation of aromatic (tocopherol and novobiocin) and olefin substrates (steroid side chains and fatty acids). Although detailed analysis of cyclopropanation, which is mechanistically related to olefin $C$-methylation, has been reported, the crystal structure of $C$-methyltransferase (C-MT), which is frequently found in the biosynthesis of various natural products, has not
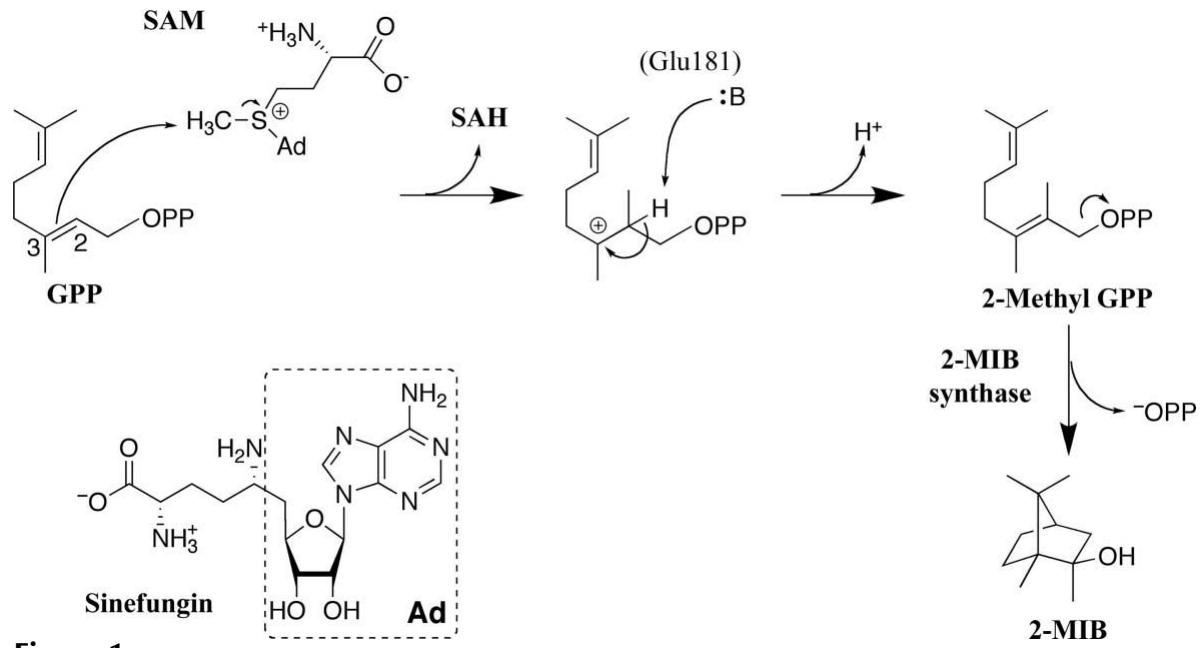

Figure 1

2-MIB biosynthesis pathway from GPP via 2-methyl-GPP. The structures of SAM and sinefungin (SFG) are also shown.

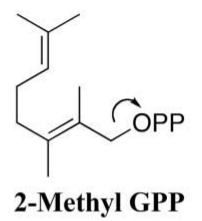

been solved to date. GPPMT is the first enzyme observed to catalyze $C$-methylation of the universal isoprenoid precursor prenyl pyrophosphate. Here, we describe the crystal structures of free GPPMT, a binary complex with the cofactor $S$-adenosyl-L-methionine (SAM) and a ternary complex with GPP and sinefungin (SFG, an SAM mimic). GPPMT shows a novel hexameric assembly in the crystal as well as in solution. We discuss the detailed mechanism of the proposed enzymatic reaction based on the ternary crystal structure revealed in complex with the natural substrate GPP and SFG by comparison with the apo form and the binary complex form with SAM.

\section{Materials and methods}

\subsection{Structure determination}

The procedures used to prepare the proteins and crystals have been described previously (Ariyawutthiphan et al., 2011). We used three types of crystals in this study, i.e. apo, a complex with SAM and a tertiary complex with both SFG and GPP (hereafter called the substrate complex), to analyze how this enzyme recognizes the substrates and its reaction mechanism. Methods for solving the structures have previously been published (Ariyawutthiphan et al., 2011); the data-collection, processing and structure-refinement statistics are summarized in Table 1. All data sets were integrated, merged and scaled using HKL-2000 (Otwinowski \& Minor, 1997). Molecular replacement was performed using MOLREP (Vagin \& Teplyakov, 2010). A molecular-replacement solution for the apoprotein crystal was found using the structure of RebM (PDB entry 3bus; Singh et al., 2008) as a search model. The sequence identity between these proteins is $22 \%$, with an additional $15 \%$ strong similarity. Final structure refinement was carried out using CNS (Brünger et al., 1998). NCS restraints were introduced for refinement of the substratecomplex form. The resolution limits of these structures were 2.2, 2.2 and $3.0 \AA$, respectively. In all crystal forms, the molecule assembles with point group 32; the molecules are crystallographically related in the $P 6_{3} 22$ forms, but have local symmetry in the $P 1$ form. SAM in the SAM-complex structure and SFG/ GPP in the substrate-complex structure were easily modelled from residual electron density around the active site. In the later stages of refinement, residual electron density close to the pyrophosphate moiety of GPP was present in all chains of the substrate-complex structure. All terpene synthases require a divalent metal ion, usually $\mathrm{Mg}^{2+}$ (Davis \& Croteau, 2000), suggesting the presence of $\mathrm{Mg}^{2+}$ in our GPPMT structure. Assigning $\mathrm{Mg}^{2+}$ at this position in all of the protein chains improved the $R$ values. Thus, the position of $\mathrm{Mg}^{2+}$ in the 
Table 1

Data-collection and refinement statistics.

Values in parentheses are for the highest resolution shell.

\begin{tabular}{|c|c|c|c|}
\hline & Apo GPPMT & GPPMT-SAM & GPPMT-GPP-SFG \\
\hline \multicolumn{4}{|l|}{ Data collection } \\
\hline Wavelength $(\AA)$ & 1.000 & 1.000 & 1.000 \\
\hline Space group & $P 6_{3} 22$ & $P 6_{3} 22$ & $P 1$ \\
\hline Unit-cell parameters $\left(\AA{ }^{\circ},{ }^{\circ}\right)$ & $\begin{array}{c}a=b=143.9 \\
c=66.7\end{array}$ & $\begin{array}{c}a=b=147.9 \\
c=66.5\end{array}$ & $\begin{array}{l}a=78.9, b=87.7, \\
\quad c=160.2, \alpha=100.0, \\
\beta=96.6, \gamma=90.9\end{array}$ \\
\hline Resolution range $(\AA)$ & $50-2.20(2.28-2.20)$ & $50-2.20(2.28-2.20)$ & $50-3.0(3.05-3.00)$ \\
\hline Total No. of observations & 317808 & 161781 & 290328 \\
\hline Unique reflections & 21196 (2059) & 22017 (2174) & 82669 (4153) \\
\hline Multiplicity & $15.0(12.7)$ & $7.4(7.3)$ & $3.5(3.5)$ \\
\hline Completeness (\%) & $99.9(100)$ & $98.8(99.6)$ & $96.3(95.8)$ \\
\hline Mean $I / \sigma(I)$ & $33.9(4.7)$ & $28.6(2.7)$ & $5.90(1.7)$ \\
\hline$R_{\text {merge }}$ & $0.07(0.421)$ & $0.06(0.424)$ & $0.118(0.359)$ \\
\hline $\begin{array}{l}\text { Wilson } B \text { factor } \dagger\left(\AA^{2}\right) \\
\quad \text { [resolution range used] }\end{array}$ & $33.9[4.0-2.2 \AA]$ & $52.8[4.0-2.2 \AA]$ & $39.5[4.0-3.0 \AA]$ \\
\hline \multicolumn{4}{|l|}{ Refinement } \\
\hline$R_{\text {work }} \ddagger / R_{\text {free }} \S$ & $\begin{array}{l}0.218 / 0.243 \\
\quad(0.275 / 0.304)\end{array}$ & $\begin{array}{l}0.261 / 0.294 \\
\quad(0.504 / 0.515)\end{array}$ & $\begin{array}{l}0.279 / 0.298 \\
\quad(0.435 / 0.434)\end{array}$ \\
\hline \multicolumn{4}{|l|}{ No. of atoms } \\
\hline Protein & 1925 & 1937 & 25514 \\
\hline Ligand/ion & 0 & 20 & 563 \\
\hline Water & 116 & 5 & - \\
\hline \multicolumn{4}{|l|}{$B$ factors $\left(\AA^{2}\right)$} \\
\hline Protein & 30.6 & 64.2 & 50.9 \\
\hline Ligand/ion & - & 71.0 & 51.3 \\
\hline Water & 36.7 & 48.1 & - \\
\hline \multicolumn{4}{|l|}{ R.m.s. deviations } \\
\hline Bond lengths $(\AA)$ & 0.008 & 0.009 & 0.004 \\
\hline Bond angles $\left({ }^{\circ}\right)$ & 1. 4 & 1.4 & 0.9 \\
\hline \multicolumn{4}{|c|}{ Ramachandran analysis $(\%)$} \\
\hline Favoured & 98.8 & 97.2 & 94.1 \\
\hline Outliers & 0.0 & 0.0 & 0.6 \\
\hline
\end{tabular}

$\dagger$ The Wilson $B$ factor was calculated by TRUNCATE (French \& Wilson, 1978). $\neq R_{\text {work }}=\sum_{h k l}|| F_{\text {obs }}|-| F_{\text {calc }}|| /$ $\sum_{h k l}\left|F_{\text {obs }}\right| . \quad \& R_{\text {free }}$ was calculated using $5 \%$ of reflections excluded from refinement. T The Ramachandran plot was calculated using MolProbity (Chen et al., 2010).

substrate-complex GPPMT structure was confirmed next to the pyrophosphate moiety when considered together with the enzymatic assay results (described below). The final $R$ and $R_{\text {free }}$ values of the crystal structures were 0.218 and 0.243 for the apo form, 0.261 and 0.294 for the SAM complex and 0.279 and 0.298 for the substrate complex. The volume of the cavities was calculated using POCASA (Yu et al., 2010) with a $2 \AA$ probe size and a $1 \AA$ unit grid. All of the figures showing protein structures were generated using PyMOL (DeLano, 2002). The atomic coordinates and crystal structure factors of geranyl pyrophosphate methyltransferase from $S$. lasaliensis in the apo form, in complex with $S$-adenosyl-L-methionine and in complex with sinefungin, geranyl pyrophosphate and magnesium ion were deposited in the PDB as entries $4 \mathrm{f} 85$ (free form), $4 \mathrm{f} 84$ (SAM complex) and 4f86 (substrate complex).

\subsection{Site-directed mutagenesis}

GPPMT mutants were generated by a two-step PCR method (Kammann et al., 1989) using the wild-type cDNA in an expression plasmid as the template. The first PCR reactions were carried out using the general forward primer and each reverse primer, as well as the general reverse primer and each forward primer, for mutagenesis. The sequences of the general primers were as follows: forward, CATGCCATGGGCAGCAGCCATCATC; reverse, CTCGAGCTACACCCGGTCGGCCG (bases in bold indicate $\mathrm{NcoI}$ and $\mathrm{XhoI}$ restriction sites, respectively). The second PCR was carried out using the forward and reverse general primers and the products of the first PCR as substrates, which were harvested after agarose gel electrophoresis. The products from the second round of PCR were harvested after agarose gel electrophoresis and digested with the restriction enzymes $N c o I$ and $X h o I$ before being introduced into the plasmid pET-28b $(+)$ (Novagen, San Diego, USA) such that $6 \times$ His was fused at the $\mathrm{C}$-terminal ends. The primer sequences used for site-directed mutagenesis are listed in Supplementary Table S1 ${ }^{1}$. The amino-acid coding regions of all mutants were sequenced using an ABI 3100 sequencer (Applied Biosystems, Foster City, USA). The expression and solubility of the recombinant proteins were confirmed by SDS-PAGE and Western blotting.

\subsection{Enzymatic assay}

$200 \mu$ reaction mixtures consisting of $1.1 \mu M$ GPPMT, $100 \mu M$ GPP and $100 \mu M$ SAM in reaction buffer $[50 \mathrm{~m} M$ Tris- $\mathrm{HCl} \mathrm{pH} 8.0$, $100 \mathrm{~m} M \mathrm{NaCl}, 20 \%(v / v)$ glycerol, $10 \mathrm{~m} M \mathrm{MgCl}_{2}, 2 \mathrm{~m} M$ EDTA, $0.2 \mathrm{~m} M \beta$-mercaptoethanol] were incubated for $2 \mathrm{~h}$. $25 \mu \mathrm{l} 0.5 M$ EDTA $\mathrm{pH} 8.0$ was then added to terminate the reaction. The reaction mixture was hydrolyzed with an excess of apyrase and acid phosphatase at $303 \mathrm{~K}$ for $2 \mathrm{~h}$. The hydrolyzed products were extracted with $n$-hexane and analyzed by gas chromatography-mass spectrometry (GCMS).

GC-MS analysis was performed using a GC-MS QP2010 apparatus (Shimadzu, Kyoto, Japan) with a DB-1 MS capillary column $(0.32 \mathrm{~mm} \times 30 \mathrm{~m}, 0.25 \mu \mathrm{m}$ film thickness; J\&W Scientific, Folsom, USA). Each sample was injected onto the column at $353 \mathrm{~K}$ in the splitless mode. After an isothermal hold at $353 \mathrm{~K}$ for $3 \mathrm{~min}$, the column temperature was increased by $20 \mathrm{~K} \mathrm{~min}^{-1}$ to $513 \mathrm{~K}$ with an isothermal hold at $513 \mathrm{~K}$ for $3 \mathrm{~min}$. The flow rate of the helium carrier gas was $1 \mathrm{ml} \mathrm{min}^{-1}$.

Enzyme-activity assays of GPPMT with other divalent cations, including $\mathrm{Zn}^{2+}, \mathrm{Ca}^{2+}$ and $\mathrm{Mn}^{2+}$, were also carried out.

\footnotetext{
${ }^{\mathbf{1}}$ Supplementary material has been deposited in the IUCr electronic archive (Reference: MH5072). Services for accessing this material are described at the back of the journal.
} 
In each case, $\mathrm{ZnCl}_{2}, \mathrm{CaCl}_{2}$ or $\mathrm{MnCl}_{2}$ was used instead of $\mathrm{MgCl}_{2}$. Enzyme activity was only observed in the presence of $\mathrm{Mg}^{2+}$ or $\mathrm{Mn}^{2+}$. Therefore, we used $\mathrm{Mg}^{2+}$ in the structure refinement.

\subsection{Gel-filtration chromatography}

Gel-filtration chromatography to check the assembly of GPPMT in solution was carried out using an S200 10/300 column (GE Healthcare, Fairfield, USA). The gel-filtration buffer consisted of $50 \mathrm{~m} M$ Tris- $\mathrm{HCl}$ pH 8.0, $100 \mathrm{mM} \mathrm{NaCl}$, $20 \%(v / v)$ glycerol, $0.2 \mathrm{~m} M \beta$-mercaptoethanol, $2 \mathrm{~m} M$ EDTA. Gel-filtration standards (Bio-Rad Laboratories, Hercules,
USA) consisting of bovine thyroglobulin ( $670 \mathrm{kDa})$, bovine $\gamma$-globulin (158 kDa), chicken ovalbumin (44 kDa) and horse myoglobin (17 kDa) were used (Supplementary Fig. S1).

\section{Results}

\subsection{Overall structure}

The final models of both apo GPPMT and GPPMT-SAM comprised 246 amino acids and 248 amino acids, respectively, of the 300 residues of the full-length protein (Fig. $2 a$ ). The first 52 residues in GPPMT-SAM and the first 54 residues in apo GPPMT could not be modelled because of a lack of significant

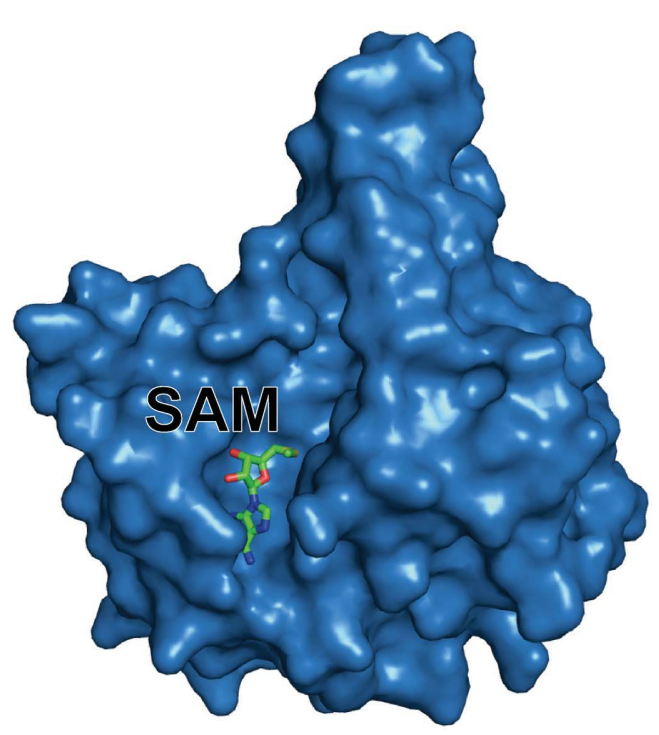

(a)

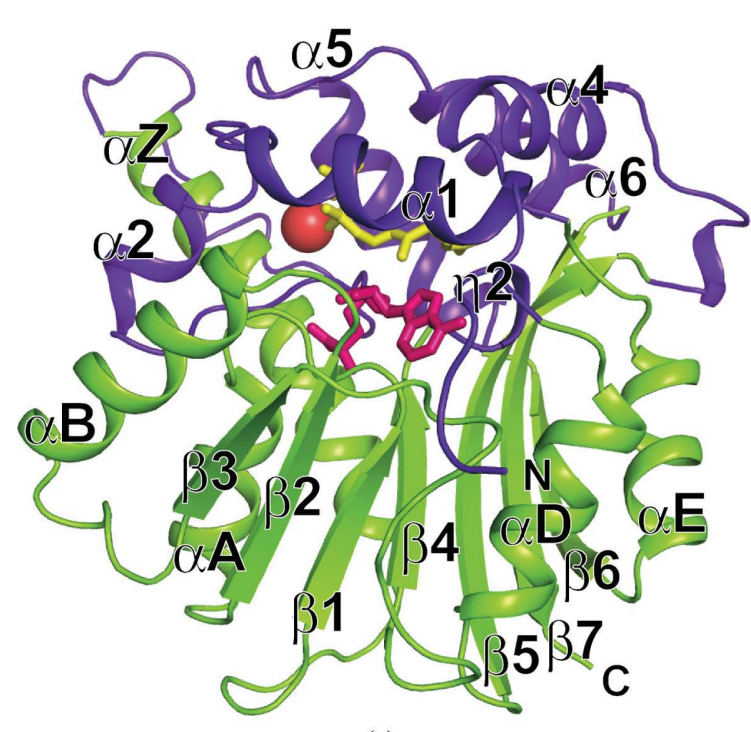

(c)

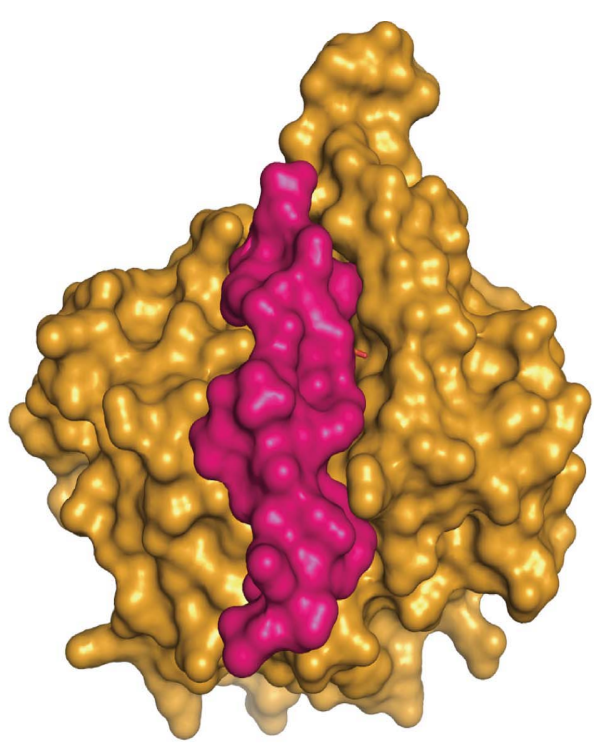

(b)

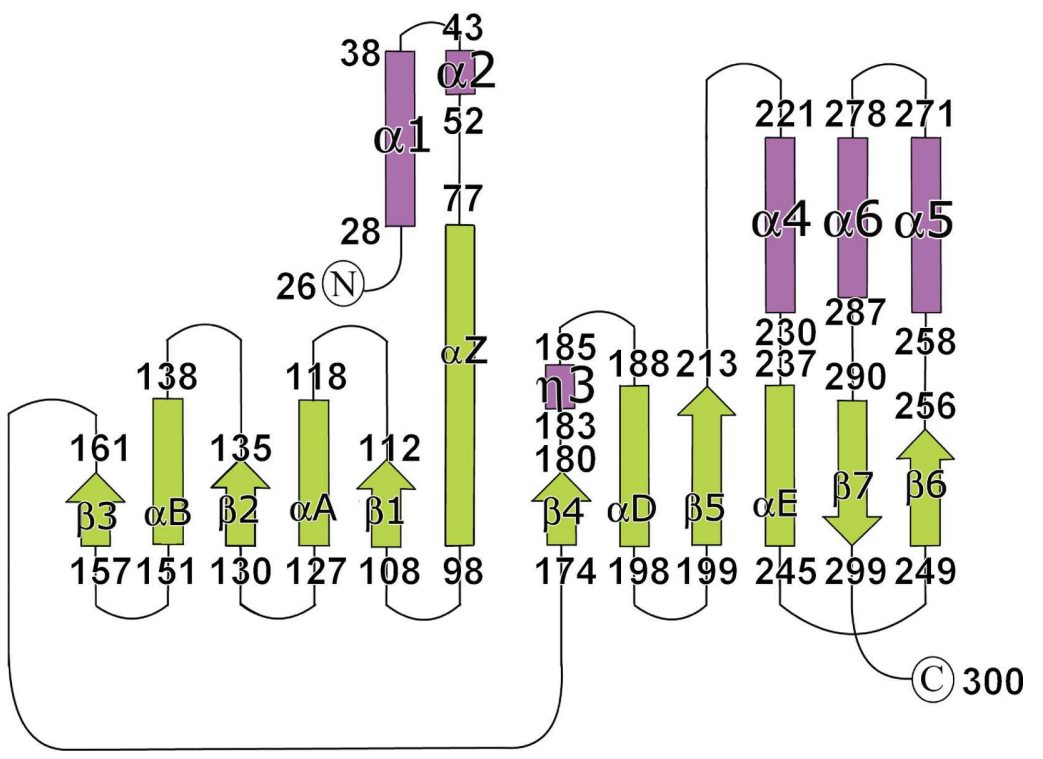

$(d)$

Figure 2

Overall structure of GPPMT. (a) Surface representation of the SAM-bound form. SAM is shown as a green stick model. (b) Surface representation of substrate-bound GPPMT. The structure of the apo form is shown in orange and the newly appearing part that is only observed in the substrate-complex structure is shown in magenta. (c) Ribbon diagram of substrate-bound GPPMT. The typical Rossmann-fold core is shown in light green and additional regions are shown in purple. SFG and GPP are shown as magenta and yellow stick models, respectively. The $\mathrm{Mg}^{2+}$ ion is shown as a red sphere. (d) Topological diagram of the substrate-bound form. The colouring is the same as that used in $(c)$. 
electron density. However, the electron density corresponding to residues 26-52 was sufficiently clear to build the model of the GPPMT-GPP-SFG structure (substrate-complex form; Fig. 2b). There is one GPPMT molecule in each asymmetric unit of apo GPPMT and GPPMT-SAM, whereas 12 molecules are present in the asymmetric unit of the substrate-complex form. Serious clashes between crystallographically related hexamers occurred if we attempted to model the N-terminal (residues 26-52) part of the apo GPPMT or GPPMT-SAM

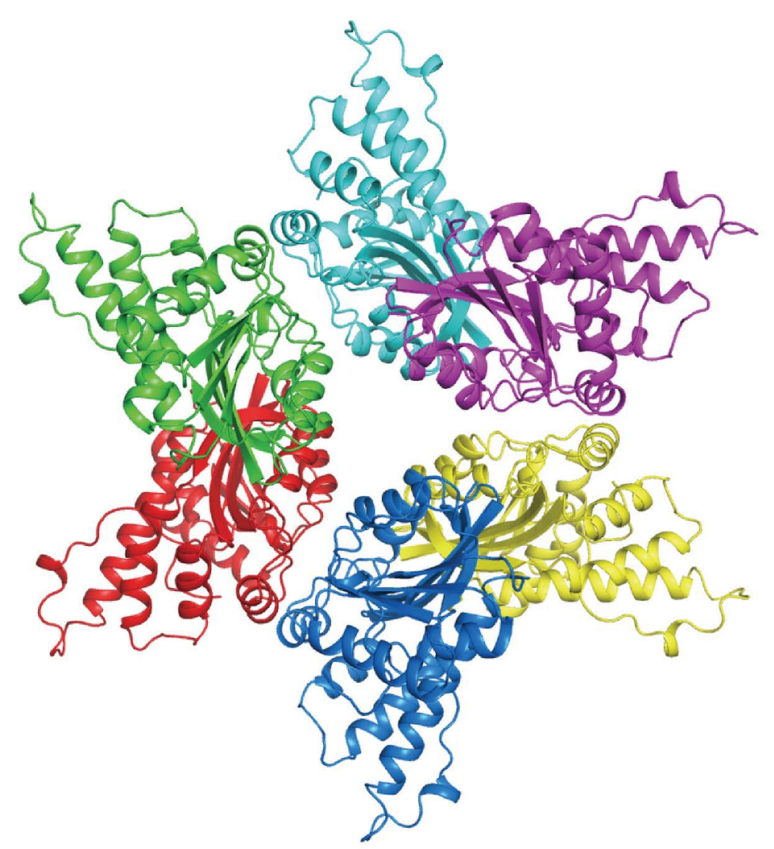

(a)

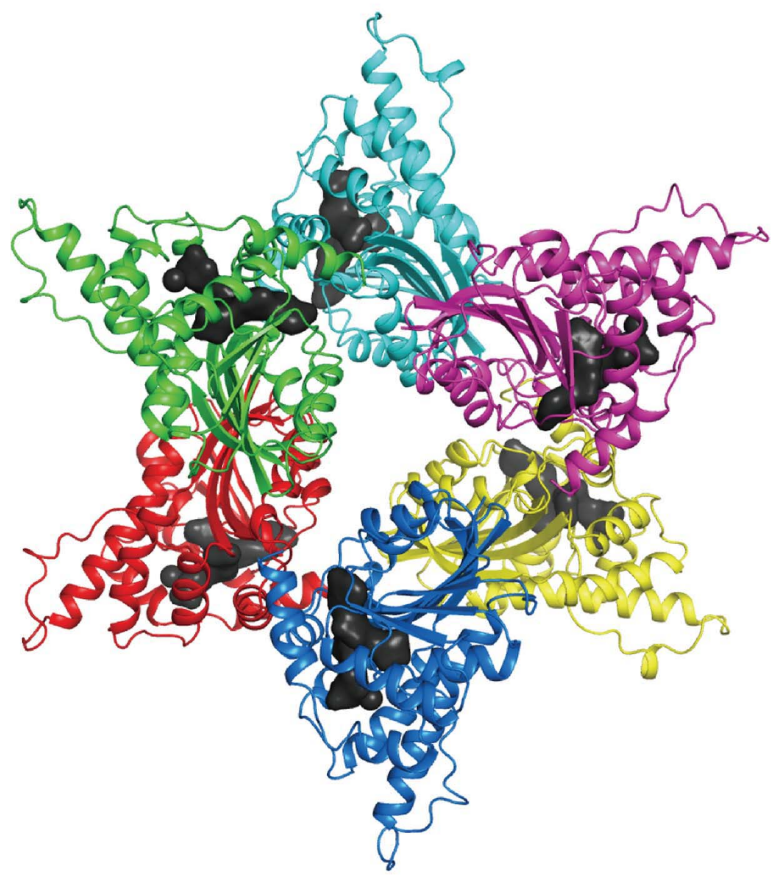

(b) structures based on the substrate-complex structure. This also supports the flexibility of the N-terminal region.

The overall structure of GPPMT consists of a mixed $\alpha / \beta$ topology with a core region containing seven-stranded $\beta$-sheets and five $\alpha$-helices (Figs. $2 c$ and $2 d$ ), which is consistent with the highly conserved Rossmann-fold core typical of SAMMTs (Martin \& McMillan, 2002; Kozbial \& Mushegian, 2005; Schubert et al., 2003). The $\beta$-strands lie in the order 3-2-1-45-7-6, with only strand 7 lying antiparallel (Fig. 2d). Additional flanking helical regions are inserted before Val51 (only in the substrate-complex structure), between Asn213 and Arg237 and between Asp256 and Phe290 (Fig. 2d). No significant structural differences were detected between the apo form and the SAM-complex form of GPPMT (r.m.s.d. of $0.34 \AA$ for $\mathrm{C}^{\alpha}$ atoms 55-300). Comparison with the substratecomplex form showed a slightly increased r.m.s.d. value (0.57 $\AA$ for $\mathrm{C}^{\alpha}$ atoms 55-300). This difference mainly arises from residues around the substrate and the $\mathrm{Mg}^{2+}$ ion in the substrate-complex structure as well as from the difference in crystal packing.

\subsection{Hexamer formation}

The structure of GPPMT is unique in terms of forming a hexameric assembly with point group 32 in both crystal forms (Figs. $3 a$ and $3 b$ ). The buried surface area between neighbouring protomers is rather extensive (1010 and $796 \AA^{2}$ in the apo crystal structure) along two independent twofold axes as calculated using PISA (Krissinel \& Henrick, 2007). There are two types of crystallographic twofold relationship and one threefold relationship in the hexamer. Specific hydrogen bonds and many specific van der Waals contacts can be observed between monomers. The inter-chain interactions of one pair include hydrogen bonds between Val254 and Val254' (3.0 $\AA$ 员 $\beta$-sheet-like interaction between crystallographically twofold-related monomers, a pair of main-chain carbonyl and amide atoms; a prime sign after the residue number indicates

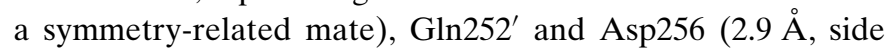
chain), Gln252' and Gln291 (3.3 ̊, side chain), and Arg237 and Asp298' (2.7 $\AA$, side chain) (Supplementary Fig. S2a). In

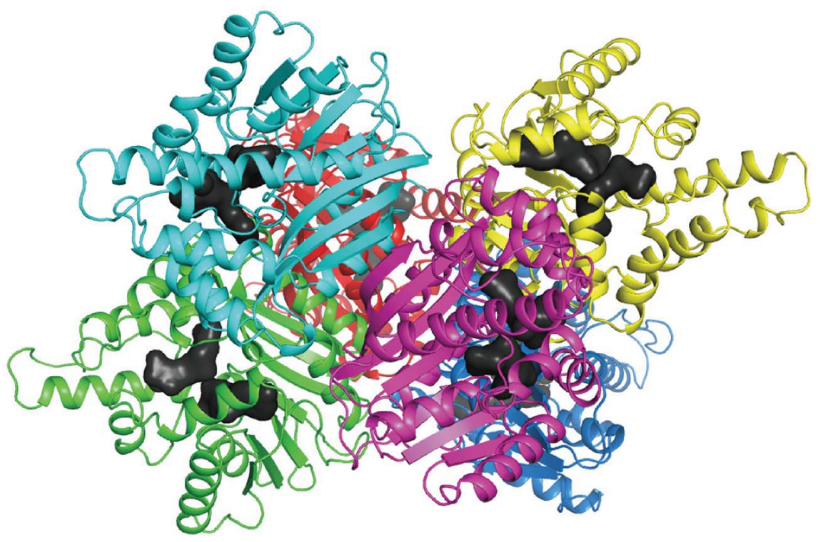

(c)

Figure 3

Ribbon diagrams of the hexameric assembly of the apo form $(a)$ and the substrate-bound form $(b)$. Each monomer is shown with different colouring. A side view of the substrate-bound form is shown in $(c)$. Sinefungin and GPP are presented as grey surface models in $(b)$ and $(c)$. 
addition to these polar interactions, significant hydrophobic interactions were observed between twofold-related monomers around the $\mathrm{C}$ atoms of residues such as Pro214, Arg238, Leu241, Ala245, Val250, Pro251, Val254 and Val300 (Supplementary Fig. S2b). With the other twofold mate (the other monomer related by the second crystallographic twofold axis), there are hydrogen bonds between Gln218 and Asn233' (2.8 $\AA$, side chain; Supplementary Fig. S2c) and Glu231 (sidechain carboxylate $\mathrm{O}$ atom) and Lys221 (3.0 ̊, main-chain amide), as well as hydrophobic interactions involving $\mathrm{C}$ atoms from residues such as Gln218, Pro219, Lys221, Ala228 and Asn233 (Supplementary Fig. S2d). Hydrogen bonds can also

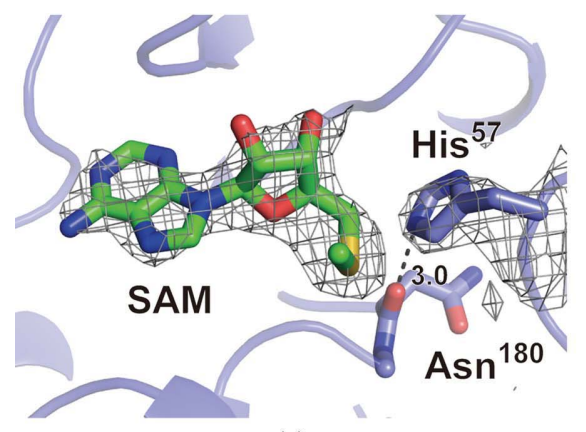

(a)

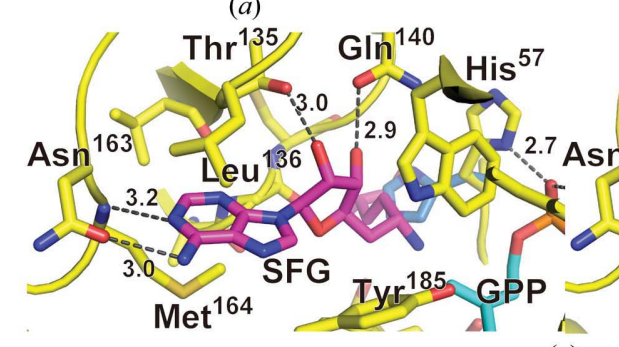

(c)

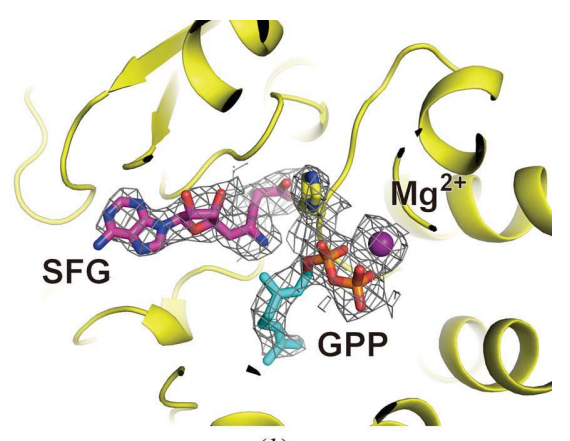

(b)
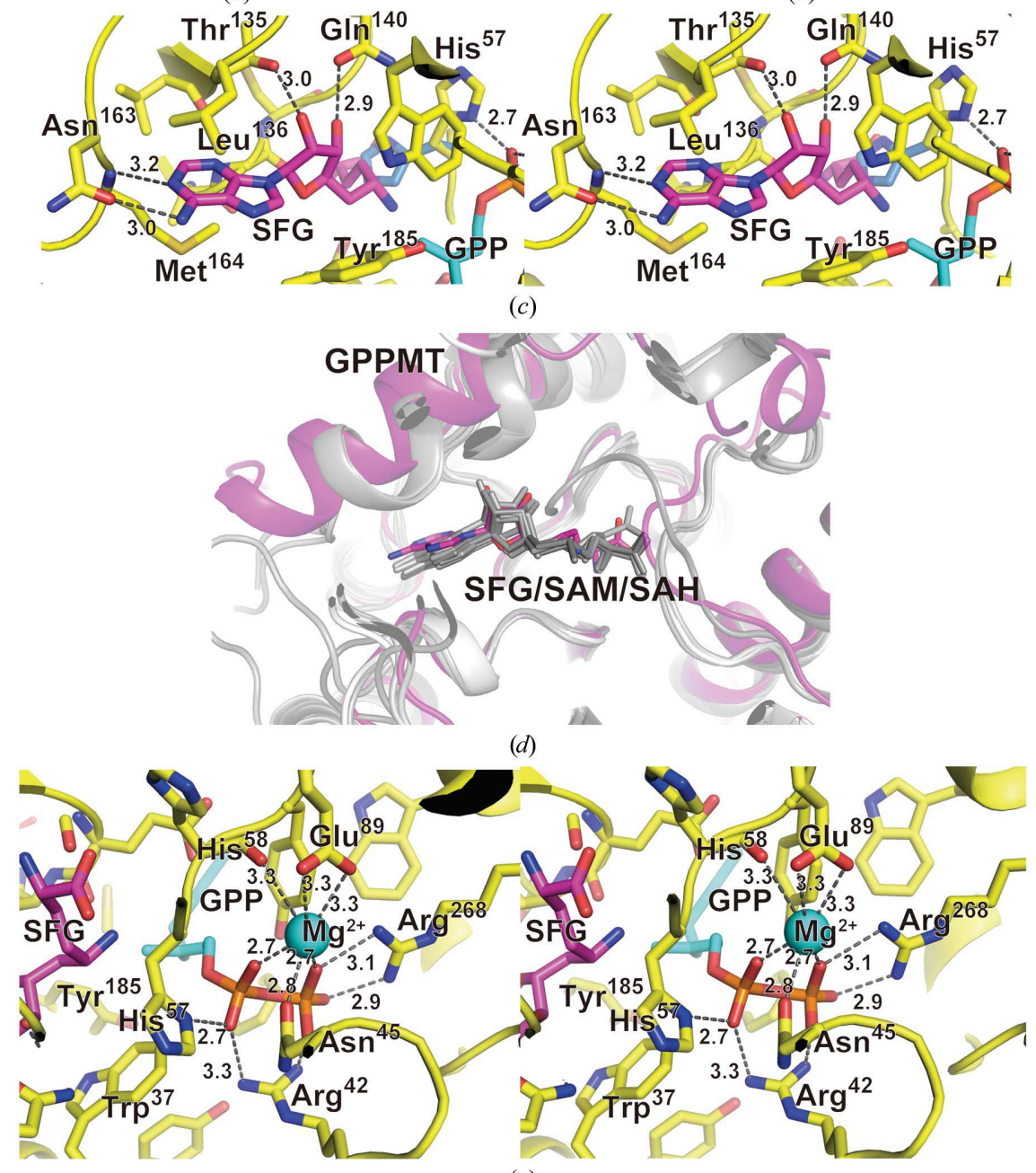

(e) be observed between threefold-related monomers; the side chains of Asp190 and Arg248' are almost in the same plane, with a proper hydrogen-bonding distance (2.9 A; Supplementary Fig. S2e).

These interactions, taken together with the observation that GPPMT forms a hexamer with local point group 32 as a substrate-complex structure, are intriguing and suggest that this enzyme uniquely acts as a hexamer. Interestingly, the $\mathrm{N}$-terminal region, which consists of residues 22-53 (which are only observed in the substrate-complex structure), makes hydrophobic interactions with a twofold-related monomer, as well as hydrogen bonds (Supplementary Fig. S2f). The residues used in these interactions are Thr27, Pro28 and Tyr29, located at the N-terminus of helix $\alpha 1$, and Tyr216', Gly217', Gln218' and Pro219' from the neighbouring monomer. Hydrogen bonds can be observed between the amide $\mathrm{N}$ atom of Tyr29 and the carbonyl O atom of Tyr216' (3.2 ^) and between the side-chain carboxyl $\mathrm{O}$ atom of

\section{Figure 4}

(a) Composite OMIT map $\left(2 F_{\mathrm{o}}-F_{\mathrm{c}}\right)$ for SAM in the SAM-complex structure. The contour level of the electron-density map is $1.2 \sigma$ and the resolution is $2.3 \AA$. SAM is shown with $\mathrm{O}$ atoms in red, $\mathrm{N}$ atoms in blue, $\mathrm{C}$ atoms in green and $\mathrm{S}$ atoms in yellow. The protein is also shown as a light blue cartoon model with the side chain of His57 (ball-and-stick representation) interacting with the main-chain carbonyl O atom of Asn180. (b) Composite OMIT map $\left(2 F_{\mathrm{o}}-F_{\mathrm{c}}\right)$ for SFG, GPP and $\mathrm{Mg}^{2+}$. The contour level of the electron-density map is $1.4 \sigma$ and the resolution is $3.0 \AA$. SFG is shown with $\mathrm{O}$ atoms in red, $\mathrm{N}$ atoms in blue and $\mathrm{C}$ atoms in magenta. GPP is shown with $\mathrm{O}$ atoms in red, $\mathrm{C}$ atoms in light blue and $\mathrm{P}$ atoms in orange. $\mathrm{Mg}^{2+}$ is shown as a purple sphere. The protein is also shown as a light yellow cartoon model with the side chain of His57 in ball-andstick representation. $(b)$ is presented from the same angle as (a). (c) Active sites of the substrate-bound form. The colours of SFG and GPP are as in $(b)$. GPPMT is shown with $\mathrm{O}$ atoms in red, $\mathrm{N}$ atoms in blue and $\mathrm{C}$ atoms in yellow. The side chain of His57 of apo GPPMT is also shown as a ball-and-stick model with $\mathrm{N}$ atoms in blue and $\mathrm{C}$ atoms in light blue. $(d)$ Comparison around the active sites of GPPMT (substrate-bound form), CmaA1 (PDB entry $1 \mathrm{kph}$ ), CmaA2 (PDB entry 1kpi), PcaA (PDB entry 111e) and Hma (PDB entry $2 \mathrm{fk} 8$ ). SFG and the protein structure of substrate-bound GPPMT are shown with $\mathrm{O}$ atoms in red, $\mathrm{N}$ atoms in blue and $\mathrm{C}$ atoms in magenta. The other proteins are presented as grey cartoon models. Cofactors (SAM or SAH) of the other proteins are also shown in grey. (e) Active-site architecture around the pyrophosphate moiety of GPP in the substrate-bound complex. Atoms are coloured as in $(c) . \mathrm{Mg}^{2+}$ is shown as a light blue sphere. 
Asp32 and the hydroxyl O atom of Tyr216 (2.8 $\AA$ ). These interactions increase the interface between the monomers (an increment of $170 \AA^{2}$ as calculated using PISA; Krissinel \& Henrick, 2007) and would fix the hexamer.

The elution volume of apo GPPMT from gel-filtration chromatography using an S200 10/300 column (GE Healthcare, Fairfield, Connecticut, USA) was between that of standard proteins with molecular weights of $670 \mathrm{kDa}$ (bovine thyroglobulin) and $158 \mathrm{kDa}$ (bovine $\gamma$-globulin) (Supplementary Fig. S1); therefore, we concluded that GPPMT forms a hexamer even in solution (hexamer molecular weight of $210 \mathrm{kDa}$ ). Although some SAM-MTs in the same fold class act as homodimers or tetramers, the majority are monomeric proteins to the best of our knowledge. In the recently reported structure of GPPMT from S. coelicolor (ScGPPMT), the corresponding residue Glu173 is proposed to play a role in stabilizing the carbocation intermediate (Köksal et al., 2012).

\subsection{SAM-binding site}

A SAM-binding site with the same fold type as in SAMMTs is present in the $\mathrm{N}$-terminal region of the $\beta$-sheet and is formed by residues from the loops following $\beta$-strands 1,2 and 3 . Some residues that recognize SAM are conserved in SAMMTs with the same fold type (Supplementary Fig. S3). The location of the SAM cofactor in the GPPMT-SAM structure was easily defined by the significant electron density corresponding to the adenosyl and sulfonium moieties (Fig. $4 a$ ), but lacked clear electron density around the methionine moiety of SAM. Therefore, we deleted this part from the refinement and the final model. In the substrate-complex structure, electron density for both SFG and GPP is unambiguous and is sufficient for discussion of the details of the substrate-recognition mechanism (Fig. 4b). The orientation of SFG is strictly determined by both hydrogen bonding and van der Waals contacts. Essentially, the recognition mode of SAM and SFG in these structures is almost identical for both the adenine and the ribose groups (Fig. 4c). The adenine group is placed in the hydrophobic milieu formed by Val134, Leu136, Met164, Tyr185 and Val186. The exocyclic N6 atom is within hydrogenbonding distance of the Asn163 carboxamide $\mathrm{O}$ atom (3.0 ; hereafter, distances are given for the $A$ chain of the substratecomplex structure). The adenine ring $\mathrm{N} 1$ makes a hydrogen bond to the main-chain amide $\mathrm{N}$ atom of Met164 (3.2 $\AA$ ). The ribose $2^{\prime}$-hydroxyl $\mathrm{O}$ atom is hydrogen-bonded to the sidechain hydroxyl O atom of Thr135 (3.0 ̊), whereas the ribose $3^{\prime}$-hydroxyl $\mathrm{O}$ atom is hydrogen-bonded to the carboxamide $\mathrm{O}$ atom of $\operatorname{Gln} 140(2.9 \AA)$. Surprisingly, the side chain of His57 is flipped in the presence of GPP to incorporate the amino-acid tail of SFG (Fig. 4c). The original orientation of the His57 side chain observed in the apo form is the same as that in the SAMcomplex form. In the substrate-complex form, the carboxylate of the SFG methionine moiety is fixed through interaction with the imidazole-ring $\mathrm{N}$ atom of His58 (2.9 $\AA$ ) and the mainchain amide $\mathrm{N}$ atom of His57 (3.3 $\mathrm{A})$.

Superimposing cofactor-bound structures of RebM (PDB entry 3bus; Singh et al., 2008), CmaA1 (PDB entry 1kph;
Huang et al., 2002), CmaA2 (PDB entry 1kpi; Huang et al., 2002), PcaA (PDB entry 111e; Huang et al., 2002), Hma (MmaA4; PDB entry 2fk8; Boissier et al., 2006) and GPPMT (this work) showed that the orientation and conformation of SAM [or $S$-adenosylhomocysteine (SAH) or SFG] are well conserved, as is the main-chain trace of the protein (Fig. 4d). In a hexameric assembly of GPPMT, six active sites are located around the outer rim and have access to the outer solvent region (Fig. 3c). Therefore, the hexameric arrangement does not seem to interfere directly with the enzymatic activity. However, interaction with GPP seems to make the hexameric assembly more stable (see §4). In the substratecomplex structure, the $\mathrm{N}$-terminal region formed by helices $\alpha 1, \alpha 2$ and loop $\alpha 2-\alpha \mathrm{Z}$ blocks access to the SAM binding site.

\subsection{Substrate recognition}

One of the distinct characteristics that are shared by all small-molecule SAM-MTs is the intrinsic N-terminal addition, which usually includes two helices (Martin \& McMillan, 2002). In the structure of substrate-bound GPPMT the N-terminal segments are composed of two helices named $\alpha 1$ and $\alpha 2$, which are connected to the structurally conserved $\alpha \mathrm{Z}$ helix by a long loop (residues 46-79; Fig. 2). These N-terminal helices, which are disordered in the apo and the SAM-bound forms, should undergo a structural rearrangement for incorporation of the substrate GPP and subsequent catalytic reaction. Helices that act as a lid for the active-site pocket have been reported in several small-molecule SAM-MTs, including RebM (Singh et al., 2008) and cyclopropane mycolic acid synthase (CmaA1; Huang et al., 2002). This region has been suggested to be flexible and to be fixed by the presence of the cofactor SAM. Notably, these N-terminal helices could not be modelled in either the apo or cofactor-bound GPPMT structures, suggesting that this part is flexible even in the presence of the cofactor SAM. From the extensive interaction with GPP, this N-terminal region is likely to be important for prenyl pyrophosphate substrate recognition. In contrast to SAM binding, substrate binding differs markedly among the smallmolecule SAM-MTs (Martin \& McMillan, 2002).

The two helices form a complete lid over the active site (Fig. $2 b$ ). The residues in this $\mathrm{N}$-terminal region, which include a few residues from a long loop between $\alpha 2$ and $\alpha \mathrm{Z}$, interact with both SAM and GPP (Figs. $4 c$ and $4 e$ ). The residues involved in interaction with the pyrophosphate moiety of GPP are mainly from the N-terminal region, such as Arg42, Asn45 and His57. Arg268, which is located in the $\alpha 5$ helix near the $\mathrm{C}$-terminus, also contributes to recognition of the pyrophosphate moiety. Additionally, Tyr59 is in close proximity to the C2 atom of GPP (3.1 $\AA$ ). We also found a magnesium ion in the active site coordinated by two $\mathrm{O}$ atoms of the pyrophosphate moiety ( $2.7 \AA$ each). Another coordination site is occupied by the $\mathrm{O}$ atom of the Asn 45 carboxamide $(2.8 \AA$ ). Two carboxylate $\mathrm{O}$ atoms from Glu89 are located at $3.3 \AA$ (Fig. 4e). A main-chain carbonyl $\mathrm{O}$ atom of His58 is also located within $3.3 \AA$ distance. In contrast, several hydrophobic residues recognize the geranyl moiety of GPP by van der 
Waals interactions, such as Tyr36, Trp37, Tyr59, Glu181 (alkyl part), Met184, Tyr185, Ile226, Phe230, Cys232, Ile234, Trp265, Phe281 and Phe290 (Fig. 4e).

\subsection{Effects of divalent cations and selected mutations}

Enzymatic activity was monitored by capillary GC-MS after enzymatic hydrolysis of the reaction mixture (Komatsu et al., 2008; Supplementary Fig. S4). To confirm which divalent ion is essential for GPPMT catalysis, enzymatic activity assays were carried out under the following conditions: with $2 \mathrm{~m} M$ EDTA and in the presence of divalent cations such as $\mathrm{Mg}^{2+}, \mathrm{Zn}^{2+}, \mathrm{Ca}^{2+}$ or $\mathrm{Mn}^{2+}$ at $10 \mathrm{~m} M$. GPPMT can convert GPP into 2-methyl-GPP in buffer containing $10 \mathrm{~m} M \mathrm{MgCl}_{2}$ (Supplementary Fig. S4), while no methylated product was detected on incubation with only $2 \mathrm{mM}$ EDTA (Supplementary Fig. S4). The enzymatic activity of GPPMT with other divalent cations, including $\mathrm{Zn}^{2+}, \mathrm{Ca}^{2+}$ or $\mathrm{Mn}^{2+}$, was also tested and activity was only observed in the presence of $\mathrm{Mg}^{2+}$ or $\mathrm{Mn}^{2+}$. The $\mathrm{Mg}^{2+}$ ion is located close to the pyrophosphate moiety of GPP in almost the opposite direction to the geranyl moiety (Fig. 4e) where methylation takes place; therefore, it is unlikely that $\mathrm{Mg}^{2+}$ participates directly in the methylation reaction. Judging from its location, $\mathrm{Mg}^{2+}$ is at least important in fixing GPP via the pyrophosphate moiety during the enzymatic reaction.

To investigate the roles of the residues around the GPP substrate, we generated mutants of Tyr59 and Glu181 because these two amino acids are closest to the $\mathrm{C} 2$ atom of the substrate GPP in our tertiary complex. We obtained Y59F and E181A mutants; however, an E181D mutant was insoluble. The Y59F mutant showed nearly comparable activity to the wild-type enzyme, whereas no activity was

Figure 5 observed for the E181A mutant. The elution volume of the E181A mutant from the gel-filtration column was the same as

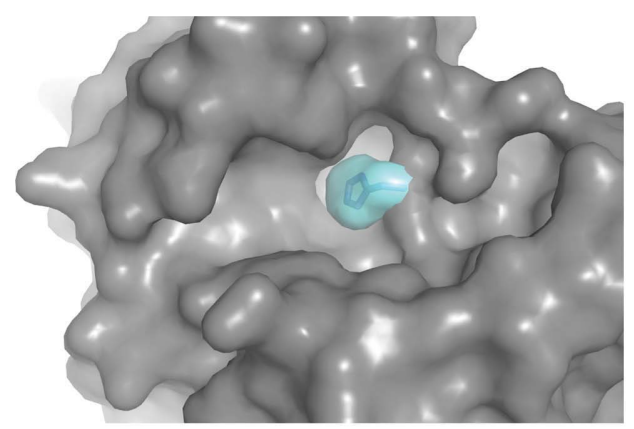

(a)

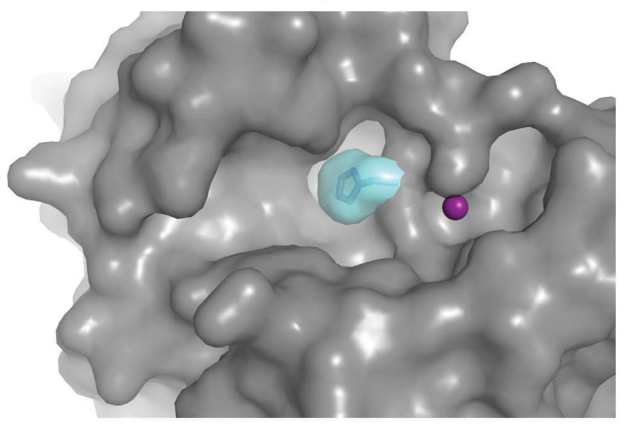

(c)

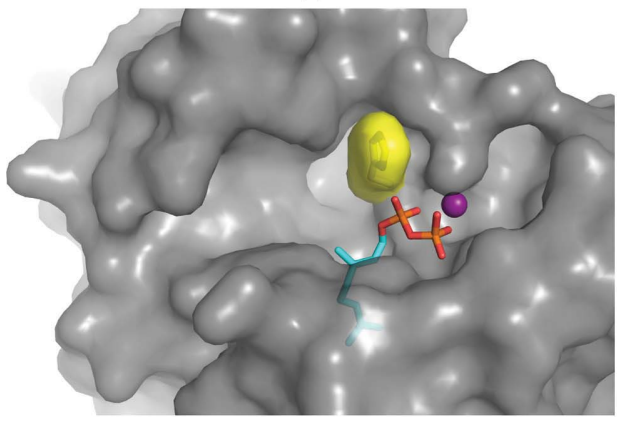

(e)

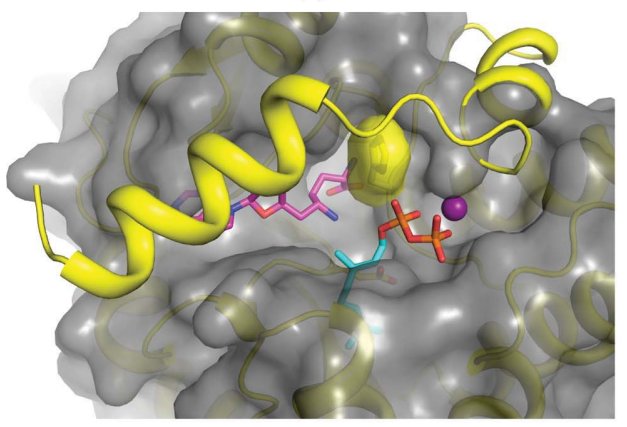

$(g)$

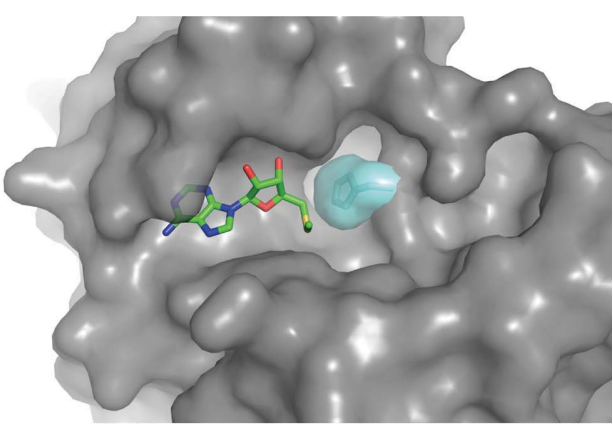

(b)

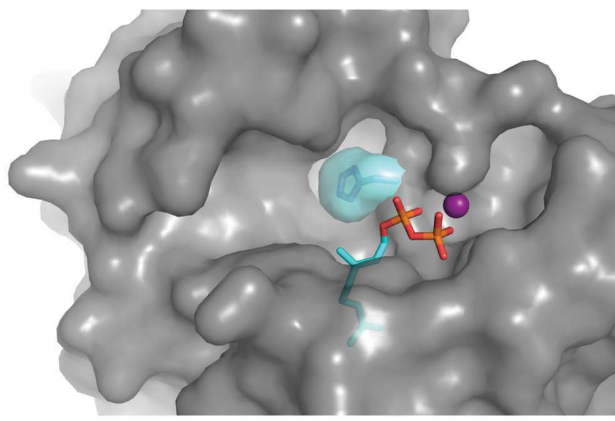

$(d)$

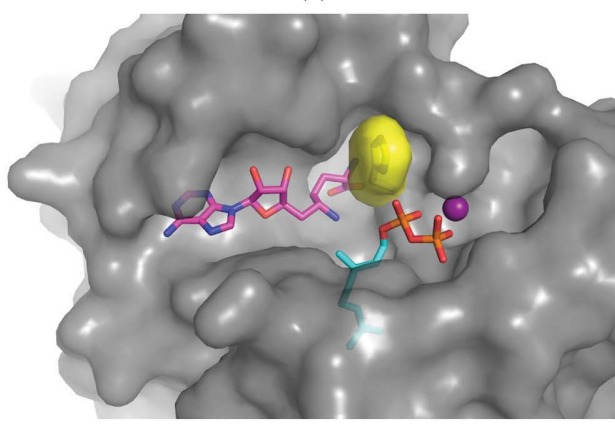

$(f)$

The active site of GPPMT as a protein-surface representation (shown in grey). (a) The side chain of His57 is shown in transparent light blue in the apo form. (b) The SAM-complex form. SAM is shown with $\mathrm{O}$ atoms in red, $\mathrm{N}$ atoms in blue, $\mathrm{C}$ atoms in green and $\mathrm{S}$ atoms in yellow. The side chain of His57 is also shown. (c) The location of $\mathrm{Mg}^{2+}$ is shown as a magenta sphere in the SAM-bound structure from superposition of the SAM-bound and substrate-bound forms. (d) The locations of $\mathrm{Mg}^{2+}$ and GPP are shown in the SAM-bound structure from superposition of the SAM-bound and substrate-bound forms. GPP is shown with $\mathrm{O}$ atoms in red, $\mathrm{C}$ atoms in light blue and $\mathrm{P}$ atoms in orange. (e) The locations of $\mathrm{Mg}^{2+}$ and GPP are shown with the orientation of His57 (shown in transparent yellow) based on the substrate-bound crystal structure. $(f)$ The locations of $\mathrm{Mg}^{2+}$, GPP and SFG are shown with the His57 side chain in the substrate-bound form. SFG is shown with $\mathrm{O}$ atoms in red, $\mathrm{N}$ atoms in blue and $\mathrm{C}$ atoms in green. $(g)$ The structure of the bound form of GPPMT is added to $(f)$ as a yellow cartoon representation. 
those of the wild type and the Y59F mutant (data not shown). Therefore, we conclude that Glu181 is a key residue for the enzymatic activity of GPPMT.

\section{Discussion}

\subsection{Ordered substrate incorporation}

We obtained crystal structures in three different states, which allows discussion of how this enzyme incorporates the two substrates (GPP and SAM) with the magnesium ion and readily catalyzes the methyltransferase reaction. The apo crystal structure has a deep cavity as the active site (Fig. 5a). The volume of the cavity for GPP and magnesium ion in the apo-crystal structure is $111 \AA^{3}$; the cavity for SAM is $59 \AA^{3}$. Cocrystal structures with SAM showed little conformational change at the active site or in the whole protein (Fig. 5b). Attempts to generate cocrystals with only GPP failed and crystals from a mixture of GPPMT and GPP gave only the apo-type structure (Ariyawutthiphan et al., 2011). However, the crystal structure of the substrate-bound form provides many insights into the incorporation of the substrate into the active site. As described previously, the methionine moiety of

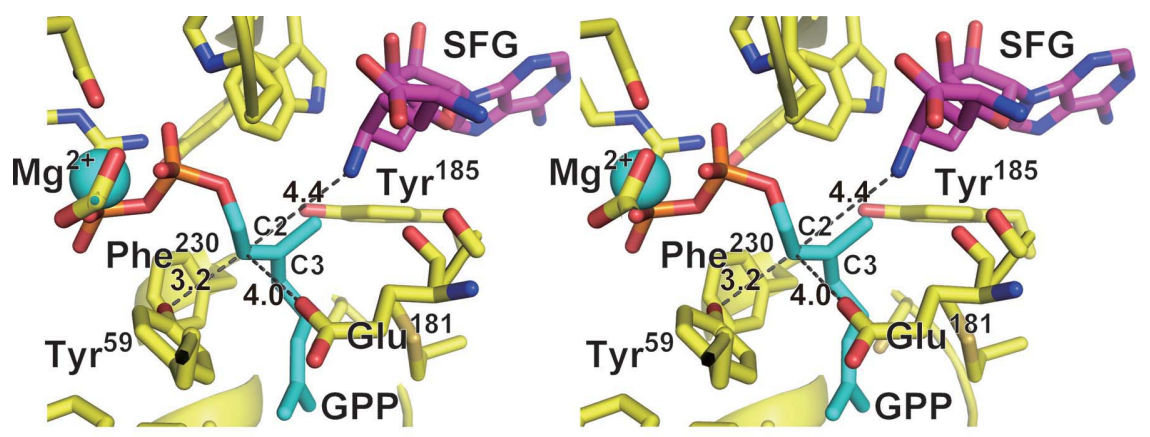

(a)

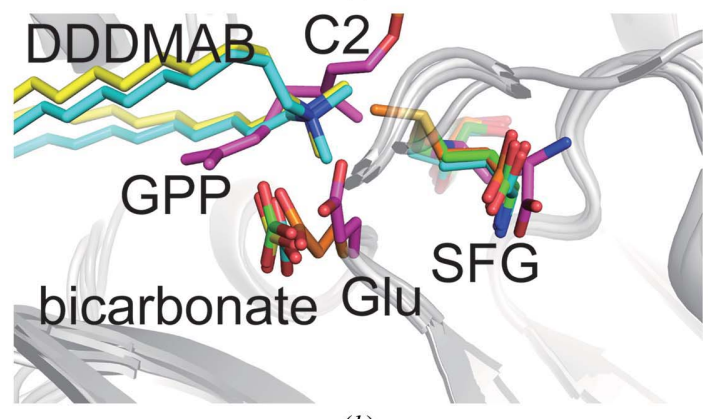

(b)

Figure 6

(a) Configuration of SFG, GPP and $\mathrm{Mg}^{2+}$ in the active site as a stereoview. Coordination of Tyr59 and Glu181 around the GPP C2 atom as well as Tyr59, Tyr185 and Phe230 facing the GPP C 3 atom are also shown. The $\mathrm{C} 2$ and $\mathrm{C} 3$ atoms are labelled. The numbers next to dotted lines indicate the distance between atoms. The same colouring is used as in Fig. 4(e). (b) Conserved bicarbonate ion and Glu residue in the active sites of GPPMT (substrate-bound form), CmaA1 (PDB entry1kph), CmaA2 (PDB entry 1kpi), PcaA (PDB entry 111e; Huang et al., 2002) and Hma (PDB entry 2fk8; Boissier et al., 2006). All proteins are shown as grey cartoons. C atoms of SFG, GPP and Glu181 of the substrate-bound GPPMT is shown in magenta. $\mathrm{C}$ atoms of $\mathrm{SAH}$, didecyldimethylammonium bromide (DDDMAB) and the bicarbonate ion of CmaA1 are shown in light blue. $\mathrm{C}$ atoms of SAH, DDDMAB and the bicarbonate ion of $\mathrm{CmaA} 2$ are shown in yellow. The $\mathrm{C}$ atom of $\mathrm{SAH}$ and the bicarbonate ion of PcaA are shown in green. The C atoms of SAM and Glu146 of Hma are shown in orange.
SAM could not be modelled. This would mean that this part is not properly placed without GPP (Figs. $5 b$ and $5 d$ ). Surprisingly, we discovered that the side chain of His57 in the strate-complex structure is flipped to open the space for SAM, Figs. $5 b$ and $5 f)$. This indicates that the binding of GPP the presence of $\mathrm{Mg}^{2+}$ ion triggers a the active site to accept SAM binding at the correct position (Figs. $5 c-5 f$ ). This process is accompanied by capping of the active site with the $\alpha$-helical N-terminal region (residues 2253; Fig. $5 g$ ). After incorporating SAM and GPP into the active site of GPPMT, the incoming methyl group of SAM interacts with the olefin and forms a new $\mathrm{C}-\mathrm{C}$ bond at $\mathrm{C} 2$, giving a carbocationic intermediate at C3 (Fig. 1).

The newly appearing N-terminal region, which consists of residues $22-53$ and is only observed in the substrate-complex many roles in the recognition of GPP (described Herer, as this part acts as a lid to the active site, this lid is completely closed. Therefore, the incorporation and arrangement of these components (GPP, SAM, $\mathrm{Mg}^{2+}$ ion and the lid) to the appropriate locations seems to be initiated by $\mathrm{Mg}^{2+}$ and GPP, followed by SAM and the lid (Fig. 5).

\subsection{Proposed mechanism of the methyltransferase}

GPPMT catalyzes olefin methylation at the C2 position of GPP. Related C-MTs are frequently involved in biosynthesis of secondary metabolites with aromatic and olefinic moieties. According to BRENDA (a web-based enzyme-information system; Scheer et al., 2011), approximately 30 members of the SAM-MTs that catalyze the $C$-methylation reaction have been identified, of which only a few have been analyzed with regard to their structural basis. To date, two reaction mechanisms for SAM-MTcatalyzed $C$-methylation have been reported. The first example is DNA methylation. A two-step direct-displacement mechanism (conjugate additionmethylation of the $\alpha, \beta$-unsaturated carbonyl system) was reported in the reaction of $C$ methyltransferase, a C5-cytosine DNA MT (Wu \& Santi, 1987). This mechanism appears to be limited to the case of substrates such as RNA and DNA, in which the olefin is conjugated with a carbonyl group (Schubert et al., 2003). The second mechanism is olefin methylation (methylation-deprotonation), which is involved in the biosynthesis of natural products such as steroids and triterpenoids. This stepwise 
mechanism via a carbocationic intermediate is more generally applicable to substrates with an isolated olefin, such as GPP.

Using SFG, a mimic of SAM, we can illustrate the moment immediately before the transfer of the methyl group. The locations of the amino-group $\mathrm{N}$ atom of SFG and the GPP C2 atom are shown in Fig. 6(a). The distance between these atoms is around $4.4 \AA$. GPPMT-mediated methylation is plausibly initiated by electrophilic attack of SAM on the GPP $\mathrm{C} 2=\mathrm{C} 3$ double bond to form a carbocation intermediate at C3 (Fig. 1). Several aromatic planes of side chains, such as Tyr59, Tyr185 and Phe230, face the C3 atom of GPP (Fig. 6a), suggesting a role in stabilization of the carbocation intermediate through cation $-\pi$ interactions. A similar situation has been observed in the active sites of mycolic acid cyclopropane synthases (MACSs; Huang et al., 2002). The $\mathrm{O}$ atoms of the side chains of Tyr59 and Glu181 are located close to the $\mathrm{C} 2$ atom, with distances of 3.1 and $4.0 \AA$, respectively (Fig. $6 a$ ). As subsequent deprotonation of the GPP $\mathrm{C} 2$ atom is required to complete the reaction, these residues might act as a general base. Mutation experiments on these two residues (Y59F and E181A) indicated that Glu181 was responsible for the final deprotonation (Supplementary Fig. S4). In addition, three sterol SAM-MTs (SMTs) are known, the substrates of which are nonconjugated olefins from the sterol-synthesis pathway: sterol 24-C-methyltransferase (EC 2.1.1.41), cycloartenol 24$C$-methyltransferase (EC 2.1.1.142) and 24-methylenesterol $C$-methyltransferase (EC 2.1.1.143). Amino-acid sequence alignment of GPPMT with these SMTs, as well as with IPPMT (described in \$4.3), showed that this glutamate residue is completely conserved (Supplementary Fig. S5). These results suggest that Glu181 is the preferred general base critical for abstraction of the $\mathrm{C} 2$ proton.

Other mechanistically related examples have been studied in the cyclopropanation of fatty acids based on crystal structures (Huang et al., 2002; Boissier et al., 2006). In these cases, methyl-group transfer from SAM to the olefin initially occurs to generate a carbocation intermediate, followed by deprotonation of the resultant methyl group to give a cyclopropane ring (Fig. 7). The difference between MT and cyclopropanase is the position of deprotonation: at the $\alpha$-proton from the carbocation in the MT reaction (Fig. 1) and at the $\beta$-proton from the carbocation in the cyclopropanase reaction (Fig. 7). Therefore, the location of the catalytic base and carbocation intermediate for deprotonation is important. In the cyclopropane-forming enzymes $\mathrm{CmaA} 1, \mathrm{CmaA} 2$ and $\mathrm{PcaA}$, residual electron density around the active sites was observed and was assigned as a bicarbonate ion located close to the cyclopropanation site (Huang et al., 2002), suggesting that the corresponding general base is a bicarbonate ion. A similar interaction of a bicarbonate ion was observed in E. coli cyclopropane fatty-acid synthase (CFAS; Iwig et al., 2005; Courtois \& Ploux, 2005). In contrast, the crystal structure of Hma (MmaA4; Boissier et al., 2006), which catalyzes the introduction of a methyl branch together with an adjacent hydroxyl group, does not contain a bicarbonate ion; however, the carboxylate group of residue Glu146 is instead located in the appropriate position. Based on these structural data, the authors speculated that Glu146 is a potential base for deprotonation (Boissier et al., 2006).

In our structures of GPPMT, the side chain of a potential base, Glu181, is located at the position corresponding to the bicarbonate ion or glutamate side chain in the loop connecting $\beta 4$ and $\eta 3$ (Fig. $6 b$ ). In the structures of $\mathrm{CmaA} 1, \mathrm{CmaA} 2$ and PcaA, the amino acid corresponding to the glutamate residue in GPPMT and Hma is glycine (Supplementary Fig. S3): a bicarbonate ion seems to be incorporated into the cavity created by the small glycine residue. Taken together, we propose that these invariant residues play similar catalytic roles (where Glu181 is equivalent to the general base) in each of these MTases for nonconjugated olefin substrates. Although crystal structures of the mycolic acid cyclopropane synthase (MACS) family have been reported, the substrate-bound structure has not been obtained because of difficulty in obtaining the actual substrate appended to an acyl carrier protein (ACP). Therefore, the structure of the tertiary complex GPPMT is most likely to reflect the actual reaction intermediate of the methyltransferase compared with that of MACS-family enzymes. In the recently reported structure of GPPMT from $S$. coelicolor (ScGPPMT), the corresponding residue Glu173 is proposed to play a role in stabilizing the carbocation intermediate (Köksal et al., 2012). This suggestion came from the fact that the side chain of Glu173 is on the same face of the $\mathrm{C} 2=\mathrm{C} 3$ double bond as the cofactor SAM and would be on the wrong face for deprotonation (Köksal et al., 2012). ScGPPMT was analyzed in complex with either SAH and GPP (PDB entry 3vc2) or with SAH and geranyl-Sthiolodiphosphate (GSSP; PDB entry 3vc1). In the structure of the complex with GPP (PDB entry 3vc2), only four monomers $(A, D, H$ and $J)$ among the 12 chains in the structure contained GPP in their active site. In the structure of the complex with GSSP (PDB entry 3vc1), all 12 monomers contained GSSP. Superimposing each monomer which harbours GPP or GSSP showed that the conformations of the prenyl side chains of each GPP and GSSP were diverse. Therefore, it is intriguing to think that GPP could move or change its conformation after accepting a methyl group to be depronated. The possibility of artifacts in the GPP conformation because of the use of SAH has also been mentioned (Köksal et al., 2012).

\subsection{GPPMT homologues in the terpenoid-modification pathway}

Although SAM-MTs that catalyze the methylation of steroid or triterpenoid side chains have been reported, there have been no reports of their being involved in catalysing the methylation of universal prenyl pyrophosphate intermediates such as IPP, DMAPP or GPP. Methylation of these intermediates could provide incorrect final products after processing. As a result, these methylated products may become inadvertent substrates for many modification enzymes because of their low substrate specificity (Koyama \& Seto, 1977). The finding that the side chain of His57 flips in the presence of the substrate GPP to appropriately accommodate 
SAM may represent the presence of a safety lock. Because His57 is one of the three consecutive His residues (56-58) that are only observed in GPPMTs (Fig. 8), the mechanism of this switching may be uniquely conserved in GPPMTs. Because of this safety lock, this enzyme would not catalyze incorrect substrates. The location of the His57 side chain which is fixed through hydrogen bonding between the imidazole $\mathrm{N}$ atom and the main-chain carbonyl O atom of Asn180 (Fig. $4 a ; 3.0 \AA$ ) in both the apo and the SAM-complex structures prevents the amino moiety of SAM being incorporated. In fact, in the SAM-complex structure no electron density was observed for the methyl moiety of SAM because of a plausible block of His57. In the substrate-complex structure, the His57 side chain interacts with an $\alpha$-phosphate $\mathrm{O}$ atom of GPP. It may be that only substrates with a phosphate moiety can release this safety lock.

To the best of our knowledge, GPPMT is the first enzyme that has been reported to catalyze this unusual methylation of prenyl pyrophosphate compounds. A putative IPPMT (termed Lon23) that could use IPP as a substrate was reported in the biosynthesis pathway of KS-505a (longestin; Hayashi et al., 2007; Supplementary Fig. S6). Its catalytic activity has recently been confirmed (unpublished work). Pairwise sequence comparison between $S /$ GPPMT and Lon 23 indicated that they share $38 \%$ sequence identity and $33 \%$ similarity at the aminoacid level (Fig. 8). Among the residues that interact with the pyrophosphate moiety in the substratecomplex structure, Asn45, His57 and Arg268 are conserved. The residues that form the hydrophobic pocket to incorporate the geranyl moiety are also conserved in the Lon 23 sequence. These residues are Tyr36, Trp37, Tyr59 (found as Phe in Lon23), Glu181 (alkyl part), Tyr185, Ile226, Phe230 (Tyr), Cys232, Ile234 (Thr), Trp265 (Phe), Phe281 (Tyr) and Phe290 (Ile). Importantly, Glu181, which has been suggested to act as a general base in this study, is also conserved in IPPMT. Furthermore, His57, which plays a key role in our safety-lock proposal, is also conserved in Lon23 (Fig. 8). GPPMT and putative IPPMT genes can be translationally coupled with genes encoding monoterpene cyclase and GGPP synthase, respectively. In general, polyprenyl synthases show a broad substrate specificity and accept artificial modified substrates. These observations indicate that methylation of common building blocks produces various unnatural methylated prenyl pyrophosphate intermediates, which may be incorporated into biologically important isoprenoids, thus increasing the risk of malfunction of living cells. Rapid trapping of methylated prenyl pyrophosphates with downstream enzymes that transform them into a specific product would avoid the formation of undesired isoprenoid derivatives. Although it is surprising that only two enzymes have been discovered in the terpenoid-biosynthesis pathway, the observations from the present study could be used to annotate new SAM-MTs that use prenyl phosphate compounds as substrates. 
We are grateful to Satoshi Omura and Haruo Ikeda (Kitasato Institute) for providing sinefungin and for helpful discussions. We also thank the beamline staff of SPring-8 (Hyogo, Japan) for technical help during the X-ray data collection. This work was supported by Grants-in-Aid for Scientific Research (22108002 to HO) from Japan Society for the Promotion of Science (JSPS). OA is supported by a scholarship from JSPS.

\section{References}

Ariyawutthiphan, O., Ose, T., Tsuda, M., Gao, Y., Yao, M., Minami, A., Oikawa, H. \& Tanaka, I. (2011). Acta Cryst. F67, 417-420.

Bentley, R. \& Meganathan, R. (1981). FEBS Lett. 125, 220-222.

Boissier, F., Bardou, F., Guillet, V., Uttenweiler-Joseph, S., Daffé, M., Quémard, A. \& Mourey, L. (2006). J. Biol. Chem. 281, 4434-4445.

Brünger, A. T., Adams, P. D., Clore, G. M., DeLano, W. L., Gros, P., Grosse-Kunstleve, R. W., Jiang, J.-S., Kuszewski, J., Nilges, M., Pannu, N. S., Read, R. J., Rice, L. M., Simonson, T. \& Warren, G. L. (1998). Acta Cryst. D54, 905-921.

Chen, V. B., Arendall, W. B., Headd, J. J., Keedy, D. A., Immormino, R. M., Kapral, G. J., Murray, L. W., Richardson, J. S. \& Richardson, D. C. (2010). Acta Cryst. D66, 12-21.

Courtois, F. \& Ploux, O. (2005). Biochemistry, 44, 13583-13590.

Davis, E. M. \& Croteau, R. (2000). Top. Curr. Chem. 209, 53-95.

DeLano, W. L. (2002). PyMOL. http://www.pymol.org.

Dickschat, J. S., Nawrath, T., Thiel, V., Kunze, B., Müller, R. \& Schulz, S. (2007). Angew. Chem. Int. Ed. Engl. 46, 8287-8290.

Eddy, S. R. (1998). Bioinformatics, 14, 755-763.

French, S. \& Wilson, K. (1978). Acta Cryst. A34, 517-525.

Giglio, S., Chou, W. K. W., Ikeda, H., Cane, D. E. \& Monis, P. T. (2011). Environ. Sci. Technol. 45, 992-998.

Gouet, P., Robert, X. \& Courcelle, E. (2003). Nucleic Acids Res. 31, $3320-3323$.
Hayashi, Y., Onaka, H., Itoh, N., Seto, H. \& Dairi, T. (2007). Biosci. Biotechnol. Biochem. 71, 3072-3081.1

Huang, C. C., Smith, C. V., Glickman, M. S., Jacobs, W. R. \& Sacchettini, J. C. (2002). J. Biol. Chem. 277, 11559-11569.

Iwig, D. F., Uchida, A., Stromberg, J. A. \& Booker, S. J. (2005). J. Am. Chem. Soc. 127, 11612-11613.

Kammann, M., Laufs, J., Schell, J. \& Gronenborn, B. (1989). Nucleic Acids Res. 17, 5404.

Köksal, M., Chou, W. K. W., Cane, D. E. \& Christianson, D. W. (2012). Biochemistry, 51, 3003-3010.

Komatsu, M., Tsuda, M., Omura, S., Oikawa, H. \& Ikeda, H. (2008). Proc. Natl Acad. Sci. USA, 105, 7422-7427.

Koyama, T., Ogura, K. \& Seto, S. (1977). J. Am. Chem. Soc. 99, 19992000.

Kozbial, P. K. \& Mushegian, A. R. (2005). BMC Struct. Biol. 5, 19.

Krissinel, E. \& Henrick, K. (2007). J. Mol. Biol. 372, 774-797.

Martin, J. L. \& McMillan, F. M. (2002). Curr. Opin. Struct. Biol. 12, 783-793.

Medsker, L. L., Jenkins, D., Thomas, J. F. \& Koch, C. (1969). Environ. Sci. Technol. 3, 476-477.

Otwinowski, Z. \& Minor, W. (1997). Methods Enzymol. 276, 307-326.

Scheer, M., Grote, A., Chang, A., Schomburg, I., Munaretto, C., Rother, M., Söhngen, C., Stelzer, M., Thiele, J. \& Schomburg, D. (2011). Nucleic Acids Res. 39, D670-D676.

Schubert, H. L., Blumenthal, R. M. \& Cheng, X. (2003). Trends Biochem. Sci. 28, 329-335.

Singh, S., McCoy, J. G., Zhang, C., Bingman, C. A., Phillips, G. N. \& Thorson, J. S. (2008). J. Biol. Chem. 283, 22628-22636.

Thompson, J. D., Higgins, D. G. \& Gibson, T. J. (1994). Nucleic Acids Res. 22, 4673-4680.

Vagin, A. \& Teplyakov, A. (2010). Acta Cryst. D66, 22-25.

Wang, C.-M. \& Cane, D. E. (2008). J. Am. Chem. Soc. 130, 8908-8909.

Wu, J. C. \& Santi, D. V. (1987). J. Biol. Chem. 262, 4778-4786.

Young, W. F., Horth, H., Crane, R., Ogden, T. \& Arnott, M. (1996). Wat. Res. 30, 331-340.

Yu, J., Zhou, Y., Tanaka, I. \& Yao, M. (2010). Bioinformatics, 26, $46-52$. 